\title{
dspace.vutbr.cz
}

\section{Comparison of Pilots' Abilities Connected with Military Flight Training using Simulation Technologies}

\author{
JIRGL, M.; JALOVECKÝ, R.
}

2021 International Conference on Military Technologies (ICMT) - Proceedings

elSBN: 978-166-543-724-0

DOl: https://doi.org/10.1109/ICMT52455.2021.9502827

Accepted manuscript

(C)2021 IEEE. Personal use of this material is permitted. Permission from IEEE must be obtained for all other uses, in any current or future media, including reprinting/republishing this material for advertising or promotional purposes, creating new collective works, for resale or redistribution to servers or lists, or reuse of any copyrighted component of this work in other works. JIRGL, M.; JALOVECKÝ, R. "Comparison of Pilots' Abilities Connected with Military Flight Training using Simulation Technologies", 2021 International Conference on Military Technologies (ICMT) - Proceedings, 2021. DOI: 10.1109/ICMT52455.2021.9502827. Final version is available at https://ieeexplore.ieee.org/document/9502827 


\section{Comparison of Pilots' Abilities Connected with Military Flight Training using Simulation Technologies}

\author{
Miroslav Jirgl \\ Department of Control and Instrumentation, \\ Faculty of Electrical Engineering and Communication \\ Brno University of Technology \\ Brno, Czech Republic \\ jirgl@vutbr.cz
}

\author{
Rudolf Jalovecky \\ Faculty of Military Technology \\ University of Defense \\ Brno, Czech Republic \\ rudolf.jalovecky@unob.cz
}

\begin{abstract}
This paper presents evaluation and consequent comparison of pilots' abilities and limitations in different stages of training. The results are based on modelling and assessing of pilots' behavior during simulated flight. For these purposes, the tests were performed with two groups of pilots - students of the first and the last study year at University of Defense. The results provide observable differences in behavioral parameters such as reaction delay, or quality of control. These parameters could be used for objective evaluation of influence of pilot training on pilot's abilities and limits.
\end{abstract}

Keywords—pilot's behavior, flight training, McRuer models, reaction delay, manual control, simulation technologies, flight simulator.

\section{INTRODUCTION}

In aviation, the human factor plays an important role, and a potential human failure could lead to poor flight performance, an accident, or even a disaster. The study of the relationships between diverse human factors and flight performance or activities related to flight safety thus embodies an important research domain. The researches in this field aims to map and predict a human/pilot behavior during various possible situations. For these purposes, flight simulators are still employed more often. [1 - 4]. The reason is that use of simulators enables, apart from obtaining information about human behavior, the possibility of continuous and deep acquisition of needed habits and their renewal. It allows unifying routine procedures while preserving the wide range of variants of dealing with different random or standard situations. The importance of the simulators lies also in their capability of simulating complex systems under various conditions and situations, with a focus on realistic interpretation [5].

Our study aims to measure, model and evaluate pilot behavior under specific conditions simulated using a flight simulator located at University of Defense. The initial hypothesis for our experiments is that the pilot's ability to control the aircraft reliably and safely increases with the training. Therefore, there is a certain opportunity to try to measure, test, or evaluate the state of pilot's ability to control the aircraft appropriately and objectively.

This paper is focused on presenting our methodology and approaches as well as to bring an example of statistical evaluation (with consequent comparison) of parameters describing pilots' abilities in different stages of flight training based on mathematical modelling and description of their behavior.

\section{ASSESSING OF PILOTS' ABILITIES AND LIMITATIONS}

The goal of our research focuses especially on experiments to support pilot behavior modeling analysis in association with military flight training, using flight simulators. The nature of this approach lies in repeating measuring the pilots' responses to a visual stimulus and consequent identification and evaluation of the pilot's behavioral model parameters, such as reaction delay or lead/lag constants as the so-called equalization part responsible for pilot's ability to adjust to the controlled dynamics and his/her attitude to control.

\section{A. Data Acquisition}

The vital element for our experiments is data acquisition. This process is carried out using measurements with real pilots on fixed-base flight simulator (located at University of Defense) equipped by X-PLANE 10 software (Fig. 1).

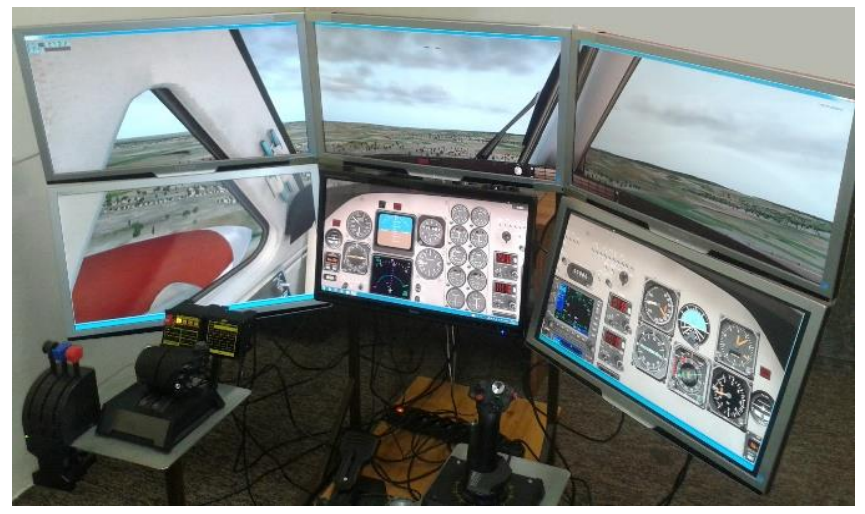

Fig. 1. The used fixed-base flight simulator at University of Defense

XPLANE-10 software enables the data recording of flight parameters during simulated flight, such as stick deflection, engine thrusts, flight velocity, flight altitude, angle of attack, etc., with sampling frequency up to $20 \mathrm{~Hz}$. The recorded data are then preprocessed and stored for consequent processing by prepared algorithms.

\section{B. Modelling and Evaluation of Pilot's Behavior}

Based on the previous studies the mathematical models derived from McRuer's Crossover Law [7] proved suitable for relatively accurate approximation of measured pilots' responses in case of simple control tasks. Especially model (1) in form of the transfer function with the gain $K$, the neuromuscular time constant $T_{\mathrm{N}}$ [s], the lag time constant $T_{\mathrm{I}}$ [s], the lead time constant $T_{\mathrm{L}}[\mathrm{s}]$, the reaction delay $\tau[\mathrm{s}]$ and the Laplace operator $s$, found application in wide area of 
experiments for approximation of human behavior connected with driving or piloting $[1,3,6,8]$.

$$
F(s)=K \cdot \frac{\left(T_{3} s+1\right)}{\left(T_{1} s+1\right)\left(T_{2} s+1\right)} \cdot \exp (-\tau s)
$$

Main advantages of using this model are especially simplicity and possibility of neurological or physiological interpretation of individual parameters connected with information processing by human and his/her corresponding control action on the elementary level (so-called control level), see Fig.2 [7, 9].

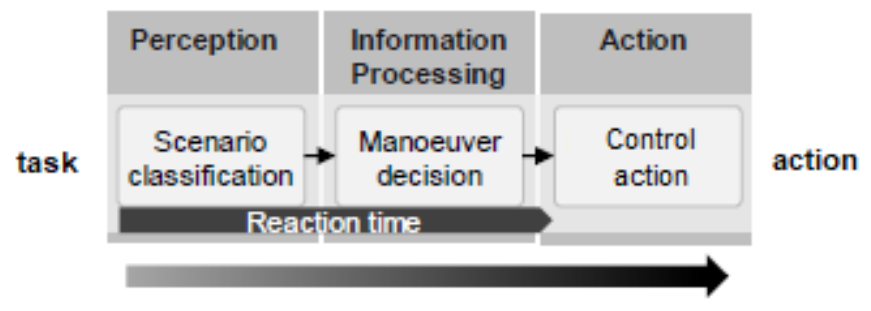

Fig. 2. The human/pilot control model [9]

One of the most limiting parameters defining human behavior is reaction time, or reaction delay $\tau$ (usually $0.1 \mathrm{~s}-1.25 \mathrm{~s}$ ). This corresponds to perception and information processing and is a crucial factor, as, in the parameter, a value too high could lead to a failure of the human control capabilities and the subsequent destabilization of the controlled system. The dynamic properties of information transfer between perception and brain are represented by the neuromuscular time constant $T_{1}$ (its value is being about $0.1 \mathrm{~s}$ ) [6 - 8].

Another aspect of human behavior model rests in the form of the control action; this process is modelled by equalization part defined by ratio of the lag time constant $T_{2}$ and the lead time constant $T_{3}$ (both about $0.1 \mathrm{~s}-10 \mathrm{~s}$ ) together with the gain $K$ [1, $6-8]$. Especially this part of the control model is responsible for the resulting form of control action and can be indirectly used for another way of pilot's behavior assessment, e.g. evaluation of quality of control. For this purpose, control theory defines quality of control criteria, such as linear, quadratic, ITAE or modified (absolute). The modified (absolute) criterion can be describe by (2) [6].

$$
J_{m}=\int_{0}^{\infty}|e(t)-e(\infty)| d t
$$

These criteria are defined as an integration of a control error $e(t)$ (where a relevant mathematical operation applied) during time in case of measuring a response of control loop to unit step change of an input signal. In real applications the infinite integration range is replaced by a finite value of measuring time.

The values of these criteria define a quality of control in view of effective or optimal setting of the regulator. A lower value of the criterion corresponds with a more optimal controller/regulator setting. In case of manual control piloting, these criteria can be applied for evaluation of pilot's ability to adapt and optimize his/her control strategy.

All of the abovementioned parameters describe dynamic properties of human/pilot, are easily identified, and can be advantageously used for evaluation of current state of a pilot in view of his/her abilities and limitations.

\section{USED APPROACH AND ALGORITHMS}

Testing of pilots on the flight simulator is performed in automatic mode controlled by developed application in MATLAB simulation environment; this application provides a detailed flight mode setting, simulator controlling, data acquisition and their storage.

The initial phase of testing lies in repeated measuring of pilots' responses to visual stimulus represented by sudden step change of altitude by $300 \mathrm{ft}$. Task of the pilots' is correction of this change to the initial altitude. This process is repeated 10 times with each pilot under the same initial conditions, namely initial altitude $H_{0}=3000 \mathrm{ft}$, speed $170 \mathrm{mph}$, angle of attack and pitch angle, including their change, was approx. 0 (note $1 \mathrm{ft}=0.305 \mathrm{~m}$ and $1 \mathrm{mph}=0.447 \mathrm{~m} \cdot \mathrm{s}^{-1}$ ). Data representing flight parameters - especially current altitude as well as a pilots' responses in form of joystick deflection, are continuously recorded with sampling frequency of $20 \mathrm{~Hz}$. The data are also sent to the instructor station with the sampling frequency of $1 \mathrm{~Hz}$ for the purposes of online monitoring and occasional correcting of the simulated flight.

During the second phase of testing the recorded data are downloaded from the storage and are mathematically analyzed. For these purposes, another developed MATLAB application is being used. The application can import and preprocess measured data and via the implemented algorithms make the analysis of pilot behavior based on identifying parameters of behavioral model (1).

\section{A. Identification Algorithm}

The identification algorithm is based on iterative optimization - MATLAB function using fminsearch simplex algorithm with a criterion function in form of the Best fit parameter defined as a mean quadratic deviation, see (3) [10].

$$
\text { Best fit }=100 \cdot\left(1-\frac{\left\|y-y_{m}\right\|}{\|y-\operatorname{mean}(y)\|}\right) \quad[\%]
$$

Where $y$ is the acquired value of the pilot's intervention (control) and $y_{\mathrm{m}}$ is the modelled (estimated) value of the pilot's intervention (control).

Because the identification process is time demanding it is carried out in form of batch process after measuring. An example of optimization (identification) results for one measured mission of one pilot is in Fig 3, where step change of altitude $H$ (red) together with a joystick deflection representing a pilot's response to this change (blue) is shown. The green curve demonstrates a result of the analysis in form of approximation of pilot's response using a model (1). Accuracy of the approximation - the Best fit value is also displayed.

\section{B. Process of Reaction Delay Obtaining}

Except above described information, Fig. 3 demonstrates a natural property of human operator (pilot) - a delay between his/her response and moment of change of input signal that should be responded. This parameter is called as reaction delay $\tau$ and it is an integral part of the human behavioral model (see model (1)). 


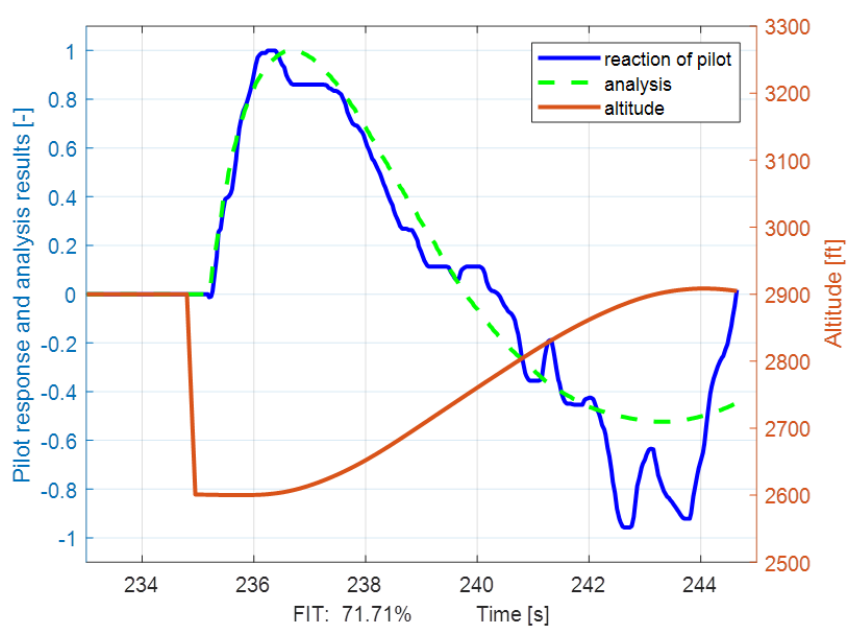

Fig. 3. An example of testing data and analysis result

The process of the reaction delay obtaining seems to be quite easy in view on the presented example. However, there are several challenging problems connected with an automatic evaluation of reaction delay, such as difficult threshold setting, or low performance of MATLAB delayest function, see [11]. These problems lead to situation where the process of the reaction delay obtaining had to be carried out manually in the past. However, the authors developed later an algorithm for reliable and fully automatic identification of reaction delay.

The algorithm for determining the optimal reaction delay, works on the principle of cyclic above described identification of the model parameters and calculation of the Best fit parameter for each mission. In each iteration, the Best fit (BF) parameter is compared to the previous highest acquired Best fit parameter (BF_last). If the current value is lower, another iteration takes place. If the current value is higher, the current model parameters and the $\mathrm{BF}$ parameter are saved in the variable BF_last and another iteration takes place.

In case of sampling frequency of $20 \mathrm{~Hz}$ corresponding to sampling period $T \mathrm{~s}=0.05 \mathrm{~s}$, the whole cycle is performed for iteration variables in the range of $2-25$. This range represents reaction delay in the unit of the number of signal samples, and for sampling period $T \mathrm{~s}=0.05 \mathrm{~s}$, the interval is $(0.1-1.25) \mathrm{s}$. This range was selected based on the interval in which the reaction delay $\tau$ is located most often.

The used approach was verified on many different data and its reliability reached about $95 \%$. A more detailed description can be found in [11].

\section{EXPERIMENT DESCRIPTION}

The algorithms and approach described above were then used for data acquired from testing subjects - students of the military pilot program at University of Defense.

Experience with flying or piloting are not required in case of new students. However, this experience is obtained during their study. During the second year of study, a theoretical background of PPL is taught as well as a skydiving training is carried out. Within the third year of study, the students are acquainted with Z-142 CAF aircraft and are beginning with practical training including approximately 65 flight hours on this type of aircraft. The fourth study year offers other 20 flight hours together with the first part of theoretical ATPL training.
In the fifth study year, the practical training is divided according to specialization - flying on L-39 and L-410 aircraft or on ENSTROM helicopter, both in sum of approx. 10 flight hours. The theoretical training in form of second part of ATPL is common for both groups. The overall experience is then about 85 flight hours for students specialized on tactical and transport flying and about 95 hours for the group focused on helicopter flying.

For the purpose of this research, the evaluation and comparison of the selected aforementioned model parameters of two group of students was performed.

The first group included 17 students of the first study year more or less without experience with flying (in view of evaluation of number of flight hours). The second group consisted of 10 students of the fifth year of study with experience that can be expressed in form of average number of 90 of flight hours.

Both groups were repeatedly tested under the same conditions via the procedure described above, i.e. measurement and evaluation of pilots' behavior during simulated flight with the task to correct the repeated sudden step changes of altitude using merely the joystick.

An example of measured data representing response of altitude to pilots' control actions (immediately after a step change of altitude) for both tested groups is shown in Fig.4.
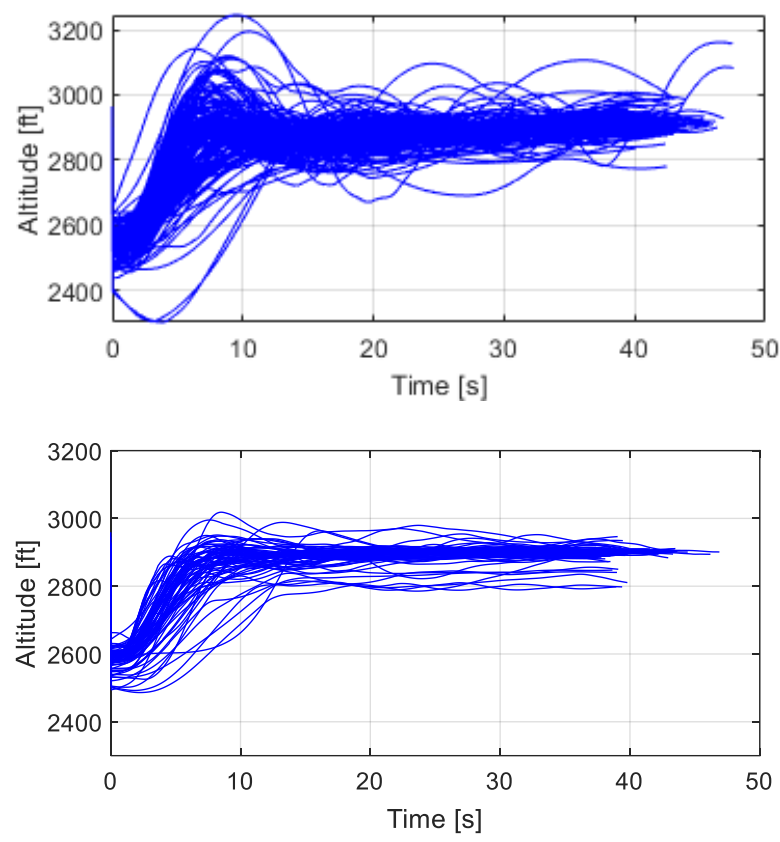

Fig. 4. Responses of altitude to pilots' control actions $-1^{\text {st }}$ group ( $1^{\text {st }}$ year students) upper, $2^{\text {nd }}$ group ( $5^{\text {th }}$ year students $)$ bottom

\section{DisCUSSION OF RESULTS}

In case of sufficient set of analyzed data, statistical evaluation of parameters describing main abilities and limitations of pilots' behavior is possible.

As stated in the previous chapter, this paper presents a results of analyses of two groups of pilots - unexperienced (students of the first study year) and trained pilots (student of the fifth study year after fully flight training). The first group-17 students were repeatedly tested, ever testing included measuring their responses on 10 consecutive step 
changes of altitude. In sum, this testing provides about 420 results. The second group including 10 students was also repeatedly tested. Total sum of the results is about 320 .

Figure 5 presents histograms and box-plots of values of reaction delay - $\tau$ for both groups of tested subjects. The outliers were neglected. The box-plots positively indicate a narrowing of dispersion of the values of the $\tau$-parameter as well as decreasing the mean value in case of more experienced pilots (students of the fifth year). That trend is observable also from the histograms. The most probable value (median) is in this case about $0.6 \mathrm{~s}$, in case of the unexperienced pilots (students of the first year) is that value about $0.8 \mathrm{~s}$. That findings correspond with theoretical expectations about the value of the $\tau$-parameter and can be interpreted as an ability of more experienced pilots to respond to sudden changes faster.
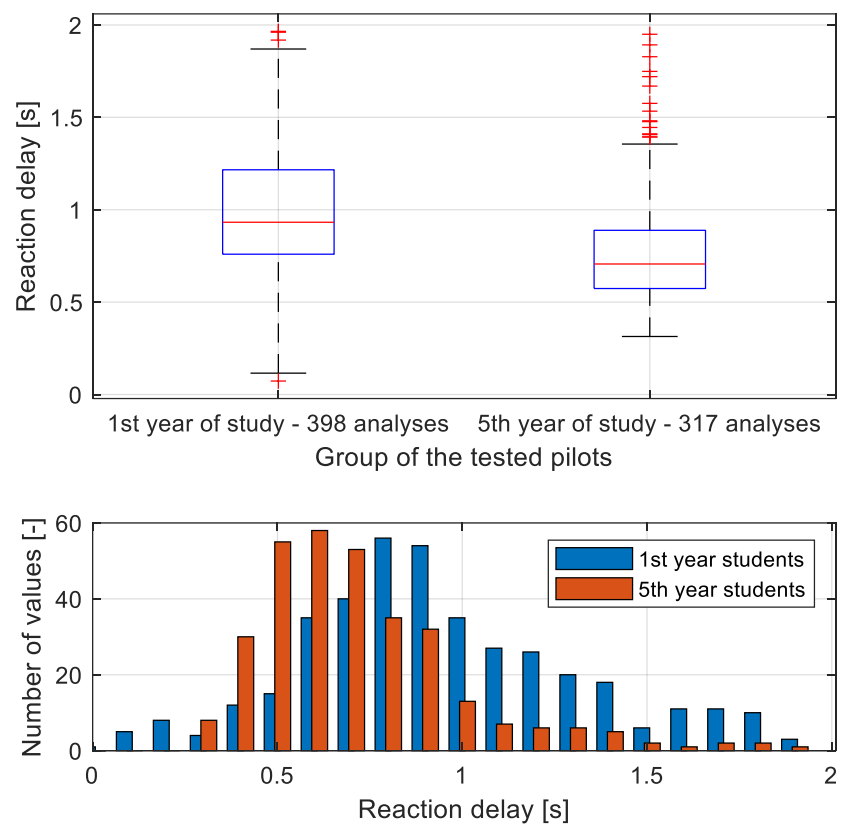

Fig. 5. Results of reaction delay - $\tau$ analysis

Results of analysis of the neuromuscular time constant $T_{1}$ are shown in Fig. 6 (outliers are neglected). This $T_{1}$ parameter represents a dynamic properties of human neuromuscular system connected with a spreading information. This explanation defines the stable and approximately constant value for each individual person and only small fluctuations of this value is possible depending on current physiological and psychic state of human $[6,7,12]$.

The results presented in Fig. 6 correspond to the described properties. The values of the $T_{1}$ parameter are in case of both tested group distributed very similarly. The medians and interval of the most probable values of $T_{1}$ are also very near for both tested groups and correspond with expected range $[6,7,12]$. That finding indicates that the values of $T_{1}$ parameter does not depend on level of training or experience.

The presented results of $\tau$ and $T_{1}$ parameters describe basic properties of tested pilots connected with their abilities or limitations. Other parameters - especially the $T_{2}$ and $T_{3}$ time constants defining the equalization part of the model (1) correspond with ability of pilot to control an aircraft (a simulated flight) where key properties are ensuring of stability and optimization.
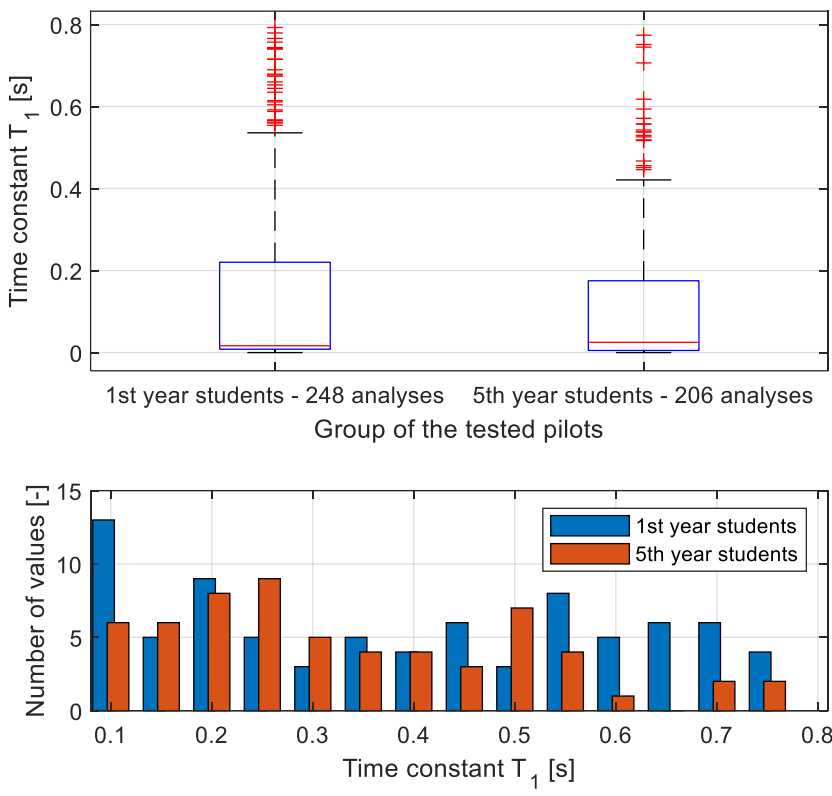

Fig. 6. Results of analysis of neuromuscular time constant $T_{l}$

Based on the McRuer's Crossover law theory, the values of $T_{2}$ and $T_{3}$ parameters and their ratio define a structure of the human/pilot regulator into the lead-lag $\left(T_{3}>T_{2}\right)$ or lag-lead $\left(T_{3}<T_{2}\right)$ configuration and are adjusted by human being according to dynamic properties of controlled element [7]. Based on this knowledge, regulator structure and expected range of these $T_{2}$ and $T_{3}$ parameters can be roughly estimated.

In spite of that valid theory, the human regulator is relatively variable and his/her setting of the equalization part of the behavioral model is to some extent individual, e.g. the value of $T_{3}$ parameter correspond with ability of prediction and fast response, in contrast to the $T_{2}$ parameter expressing a certain inertia or lag as well as an ability of damping of a fast control action [7]. In view of that properties, the values of both parameters (especially $T_{2}$ ) should be a little higher for the experienced pilots.

Medians of the $T_{2}$ and $T_{3}$ parameters evaluated from analyses focusing on identification of model (1) parameters are shown in Table 1.

TABLE I. MEDIANS OF THE $T_{2}$ AND $T_{3}$ PARAMETERS

\begin{tabular}{|l|c|c|}
\hline \multicolumn{1}{|c|}{ Group } & $\boldsymbol{T}_{2}[\mathrm{~s}]$ & $\boldsymbol{T}_{3}[\mathrm{~s}]$ \\
\hline $1^{\text {st }}$ group - unexperienced pilots & 1.1 & 5.5 \\
\hline $2^{\text {nd }}$ group - experienced pilots & 1.5 & 4.8 \\
\hline
\end{tabular}

The results presented in Table 1 defines in both cases a structure of the model (1) as lead-lag. These parameters are also related to resulting response of controlled element in form of altitude changes presented in Fig. 4. While the first (unexperienced) group respond more rapidly with higher overshoot, the responses of the second (experienced) group are calmer and without observable overshoots.

Source data representing altitude can be also used for evaluation of control quality. The control error $e(t)$ represents a difference between a required value of altitude (3000 ft) and a current value of altitude $H$. Values of $e(t)$ thus obtained are then standardized to the range from -1 to 1 and 
applied for calculation of $J_{m}$ according to (2). The results of $J_{m}$ (without outliers) are shown in Fig. 7.

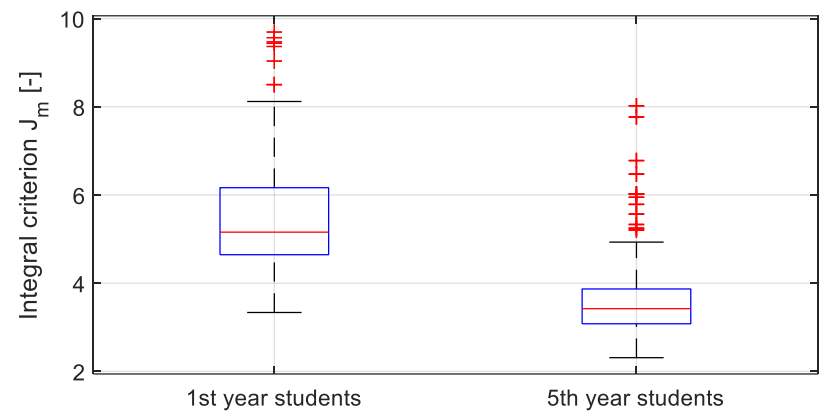

Groups of the tested pilots

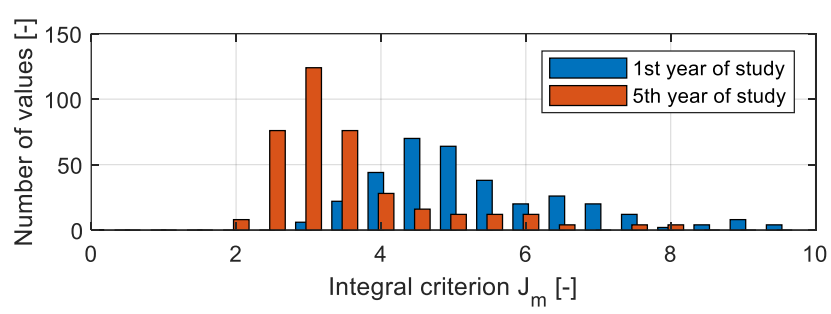

Fig. 7. Modified (absolute) criterion of quality of control

Mostly occurred values of $J_{m}$ integral criterion are lower in case of the experienced pilots. These results support all above presented results and conclusions as well as correspond with waveforms of altitude presented in Fig. 4.

\section{CONCLUSION}

The goal of this paper was to demonstrate an approach for evaluation of pilots' abilities and limitations and consequently to compare results of two groups of pilots in different stages of training.

The used approach is based on methods known from control theory and system engineering, i.e. mathematical modelling and identifying parameters describing pilots' behavior from the view of dynamic properties. Input data for this experiment was acquired via testing procedure on fixedbase flight simulator at University of Defense where simple testing task was defined and implemented.

Testing procedure was applied to two groups of military pilots. The first group included 17 unexperienced pilots students of the first study year without flight training. The second group consisted of 10 students of the fifth year of study with experience that can be expressed in form of average number of 90 of flight hours.

The achieved results presented in this paper provide observable differences in behavioral parameters such as reaction delay, quality of control or other parameters. Although the tested groups represent only limited and not very statistically significant set of data, the results indicate the used approach could be an effective tool for an objective assessment of pilots' training level.

Future work will be focusing on classification of data measured yet as well as continuation in testing other pilots. The effort of the authors is concentrated on evaluation of a broader and statistically significant set of data based on presented approach for the purpose of generalizing the presented conclusions.

\section{ACKNOWLEDGMENT}

The work presented in this paper has been supported by the Czech Republic Ministry of Defense-University of Defense development program "AIROPS - Conducting aerospace operations".

This research was also supported by the grants "Research in Automation, Cybernetics and Artificial Intelligence within Industry 4.0 " financially supported by the Internal science fund of Brno University of Technology, grant number FEKTS-20-6205; and SECREDAS Product Security for Cross Domain Reliable Dependable Automated System, H2020ECSEL, EU, grant number 783119-1.

\section{REFERENCES}

[1] M. M. Lone and A. K. Cooke, "Pilot-model-in-the-loop simulation environment to study large aircraft dynamics", Proceedings of the Institution of Mechanical Engineers, Part G: Journal of Aerospace Engineering, vol. 227, no. 3, pp. 555-568, Jan. 2012.

[2] Z. Slanina, S. Mikolajkova, and D. Vala, "Human vehicle interaction", Proceedings of the Institution of Mechanical Engineers, Part G: Journal of Aerospace Engineering, vol. 227, no. 3, p. 020050-, Jan. 2012.

[3] R. A. Hess, F. Marchesi, and D. Vala, "Analytical assessment of flight simulator fidelity using pilot models", Journal of Guidance, Control, and Dynamics, vol. 32, no. 3, pp. 760-770, 2009.

[4] Y. Zhang, S. McGovern, "Mathematical models for human pilot maneuvers in aircraft flight simulation", ASME International Mechanical Engineering Congress and Exposition; ASME: New York, NY, USA, 2010; Volume 13, pp. 35-39.

[5] D. J. Allerton, F. Marchesi, and D. Vala, "The impact of flight simulation in aerospace", The Aeronautical Journal, vol. 114, no. 1162, pp. 747-756, 2010.

[6] M. Jirgl, J. Boril, and R. Jalovecky, "Statistical evaluation of pilot's behavior models parameters connected to military flight training", Energies, vol. 13, no. 17, pp. 747-756, 2020.

[7] D. T. McReuer and E. S. Krendel, Mathematical models of human pilot behavior, AGARD AG-188, 1974.

[8] M. Mulder, D. M. Pool, D. A. Abbink, E. R. Boer, P. M. T. Zaal, F. M. Drop, K. van der El, and M. M. van Paassen, "Manual control cybernetics: state-of-the-art and current trends", IEEE Transactions on Human-Machine Systems, vol. 48, no. 5, pp. 468-485, 2018.

[9] C. Roesener, M. Harth, H. Weber, J. Josten, L. Eckstein, F. M. Drop, K. van der El, and M. M. van Paassen, "Modelling human driver performance for safety assessment of road vehicle automation: stateof-the-art and current trends", 2018 21st International Conference on Intelligent Transportation Systems (ITSC), vol. 48, no. 5, pp. 735-741, 2018.

[10] L. Ljung, "Experiments with identification of continuous time models", in IFAC Proceedings Volumes, 2009, vol. 42, no. 10, pp. 1175-1180.

[11] M. Jirgl, J. Boril, and R. Jalovecky, “Automated search and evaluation of pilot's reaction delay", in 2019 International Conference on Military Technologies (ICMT), 2019, vol. 42, no. 10, pp. 1-5.

[12] R. D. Campbell and M. Bagshaw, Human performance and limitations in Aviation, 3rd. Wiley-Blackwell, 2002. 